\title{
The Active Effect of Electric Field on Heat Transfer Enhancement for Spray Cooling
}

\author{
Haojie $\mathrm{Xu}^{1}$, Junfeng Wang ${ }^{\star 1}$, Dongbao Wang ${ }^{1}$, Wei Zhang ${ }^{1}$, Jiang Yao ${ }^{1}$ \\ ${ }^{1}$ School of Energy and Power Engineering, Jiangsu University, Zhenjiang, China \\ *Corresponding author email: wangjunfeng@ujs.edu.cn
}

\begin{abstract}
Spray cooling is confirmed an efficient route for high heat flux removal with numerous droplets impinging onto the heated surface. By means of high applied potentials, the spraying liquid would be elongated into cone or jet producing ultrafine droplets, further resulting in heat transfer enhancement. This paper mainly concerned the effect of electric field on electrospray (ES) morphology and the subsequent cooling performance in terms of spray characteristics, liquid breakup mode transition, and droplets impinging behaviour. The results showed that with the increase of applied voltage, the spray droplets size significantly decreased due to the weakened surface tension caused by charge concentration while the electrohydrodynamic force accelerated the speed of droplets impacting the hot substrate. In addition, the charged droplets was found to spread with thinner liquid film in single phase region while its rebound from the surface was restrained by image force in film boiling region. Specifically, ES demonstrated a maximum enhancement rate of 2.8 times over neutral (no voltage) cooling. Overall, ES cooling could significantly improve heat transfer performance varying with spray modes and droplets behaviour, and the outcome would contribute to a better understanding of the enhancing mechanism of spray cooling by electric field.
\end{abstract}

\section{Keywords}

Electrohydrodynamic; Electrospray, Spray cooling; Heat transfer

\section{Introduction}

Spray cooling is a technique in which a liquid is fully pressurized before being atomized into droplets through a nozzle, then rapidly ejected to a hot surface, which removes heat with droplet impact, film motion, bubble formation, and environmental convection [1]. Because of its efficient heat removal, this technique has been widely utilized in various fields, such as metallurgy heat treatment, space thermal control, integrated circuit heat management, and biomedicine laser operation [2]. Over the past few years, increasing research has been conducted on the heat transfer mechanism, influence factors, and enhancement of spray cooling [3]. The ES process was first theoretically studied by Rayleigh in 1882 . The formation of charged droplets is shown in Figure 1(a). High voltage is applied to the capillary to charge the working liquid with like charges, which is mostly distributed on the surface of the liquid. The charged liquid drops from the meniscus tip (also called Taylor cone [4]) once the electric force and gravity overcome surface tension. Various breakup modes of liquid flow have been defined, such as dripping mode, spindle mode, oscillating mode, cone-jet mode, multi-jet mode, and ramified mode. Four stable mode morphologies are shown in Figure 1(b) and will be further discussed in the subsequent sections. The evolution of these modes is accompanied by a change in spray characteristics, including droplet diameter, droplet velocity, spray angle, and penetration distance, which we hypothesize to have a crucial effect on the heat transfer of spray cooling.

The multiphase flow and heat transfer mechanisms involved in ES cooling are very complicated due to the coupling effects of the electric field, temperature field, and flow field. 
Until now, little research has been conducted on this subject, and the ES heat transfer mechanism is still unclear. The electric field was first applied to spray impingement heat transfer by Feng [5], who studied the effects of applied voltage, flow rate, spray spacing, and heated surface geometry and found that the enhancement rate was 1.7 times at a low heat flux under certain conditions. Deng [6] obtained a high heat flux of $96 \mathrm{~W} / \mathrm{cm}^{2}$ with ethanol as the coolant. Throughout the previous literatures, ES has shown promise in spray cooling, and some fundamental research has been conducted. However, correlations between the spray modes and heat transfer performance for ES cooling have not been adequately studied, and the ES cooling technique is not well understood, especially for precise cooling. The major contribution of our work is to characterize the effects of spray mode evolution on heat transfer performance, which previous research does not consider. First, the spray characteristics of four different spray modes are studied. We consider droplet size, spray velocity, repetition frequency, and spray swath, which are used to analyze the difference in cooling performance as spray mode switches. Then, the cooling capacities of different ES modes are quantitatively investigated, and the enhancement ratio of ES to neutral spray cooling is specified. In addition, we visualize the impact behaviour of spray droplets on the heated surface to explain the heat transfer performance of ES with different modes. Our study reveals the enhancement mechanism of ES cooling for more precise control.

(a)

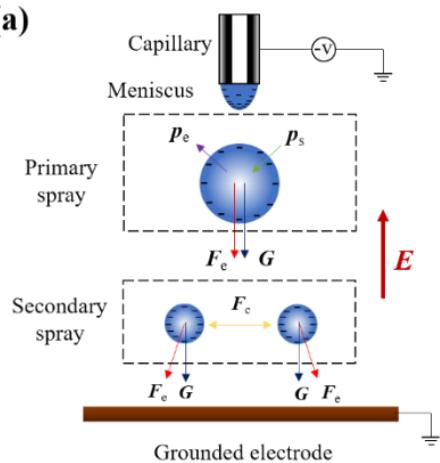

(b)

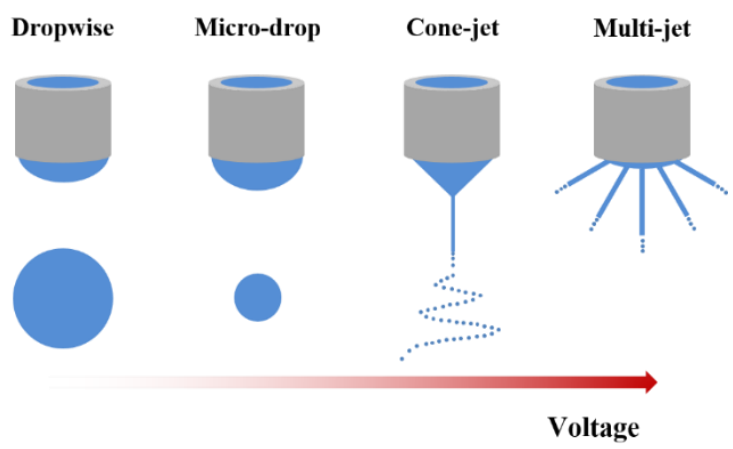

Figure 1. Schematic of (a) the force acting on ES droplets and (b) four typical ES modes, where $p_{e}$ is the static pressure difference due to surface tension, $p_{\mathrm{s}}$ is the expansive force due to Coulomb force, $G$ is the gravity, $F_{\mathrm{e}}$ is the electric field force and $F_{\mathrm{c}}$ is the Coulomb repulsion between droplets.

\section{Experimental setup and Methods}

As shown in Figure 2, the experimental setup consists of three primary components, including an electrospray section, a heat transfer section, and a visualization section.

Ethanol is employed as the coolant due to its excellent charge and heat transfer characteristics. The fluid is supplied to a stainless steel capillary with an inner diameter of $0.2 \mathrm{~mm}$ and an outer diameter of $0.4 \mathrm{~mm}$ via a syringe pump (KDS-100). A high negative voltage DC power supply (voltage $0-30 \mathrm{kV}$, current $0-2 \mathrm{~mA}$ ) is connected to the capillary, forming a non-uniform pin-to-plate electric field to the grounded copper heated surface. The space between the capillary nozzle and counter electrode is set to $10 \mathrm{~mm}$, the flow rate ranges from 5 to $30 \mathrm{ml} / \mathrm{h}$, and the applied voltage reaches up to $6.8 \mathrm{kV}$.

The heat transfer block is made of copper, which is heated by an alumina ceramic heater with a maximum power of $100 \mathrm{~W}$. The heating element is placed inside the copper block, bonded with thermal grease. Meanwhile, the copper block is well coated by glass fibre insulation to reduce heat loss to the environment, except at the top test surface. A proportion integration differentiation (PID) controller is used to avoid overheating. The size of the heat transfer 
surface is $20 \mathrm{~mm} \times 20 \mathrm{~mm}$, and it is treated with 2000 grit abrasive paper for each test. Three $\mathrm{K}$ type thermocouples are put vertically on the centreline at distances of $5 \mathrm{~mm}, 10 \mathrm{~mm}$, and $15 \mathrm{~mm}$ from the top surface. The temperature data is recorded with an Agilent data acquisition instrument (34970A) at a frequency of $1 \mathrm{~Hz}$.

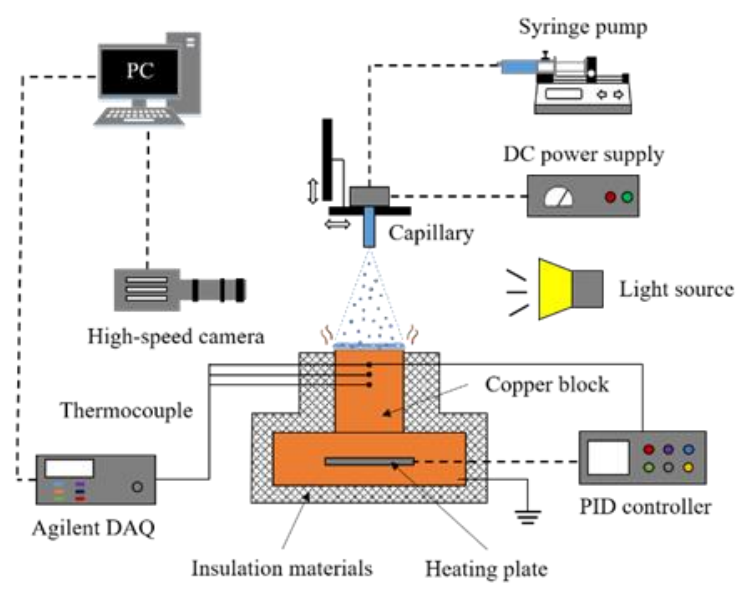

Figure 2. Schematic of the ES cooling setup.

Details of ES droplet behaviour are captured by a high-speed camera (Phantom V1611) matched with a microscopic zoom lens (Navitar 12-X). A LED cold light source is placed opposite the camera for backlit shooting. Because the spray process requires a high frame rate to capture, especially with high applied voltage, the camera is set to work at $1024 \times 768$ pixels and a sample rate of $4000 \mathrm{fps}$. Additionally, a Nikon DSLR camera (D5 XQD) and a telephoto zoom lens (AF Micro Nikkor $200 \mathrm{~mm}$ ) are utilized to better show the morphology of ES cooling.

All experiments are operated in ambient conditions of $25^{\circ} \mathrm{C} \pm 0.5^{\circ} \mathrm{C}$, with the copper block temperature ranging from $30^{\circ} \mathrm{C}$ to $150^{\circ} \mathrm{C}$. The heat transfer near the testing surface is assumed to be a one-dimensional process on account of the good insulation treatment and slender shape design of the heated copper block. The heat flux $q$ can be calculated by Fourier's law, which is described as:

$$
q=k_{c u} \frac{\Delta T}{\Delta x}
$$

where $k_{\mathrm{cu}}$ is the thermal conductivity of copper $(401 \mathrm{~W} /(\mathrm{m} \cdot \mathrm{K})$ in this paper), and $\Delta \mathrm{T}$ and $\Delta \mathrm{x}$ represent the temperature difference and spacing $(5 \mathrm{~mm}$ in this paper) of two adjacent thermometers.

The surface temperature $T_{\mathrm{s}}$ and convection heat transfer coefficient $\mathrm{h}$ can be defined as:

$$
\begin{aligned}
& T_{S}=T_{1}-\Delta T \\
& h=\frac{q}{T_{S}-T_{\text {liq }}}
\end{aligned}
$$

where $T_{1}$ is the measured temperature of the first layer ( $5 \mathrm{~mm}$ from the testing surface), and $T_{\text {liq }}$ is the inlet temperature of working fluid.

\section{Results and Discussion}


Electrohydrodynamics (EHD), a relatively new branch, is the combination of fluid mechanics and electrodynamics. Multi-component forces act on liquids, resulting in many interesting phenomena, such as electrowetting, electrospray, and corona winds. Typically, the ES process is accompanied by complex deformation, breakup, coalescence, and separation of charged droplets. Spray modes will vary with the applied EHD force, and their classification has been well investigated [7].

Here, four different ES modes of ethanol with a flow rate of $20 \mathrm{ml} / \mathrm{h}$ are shown in Figure 3. It can be observed that the dropwise, micro-dripping, cone-jet, and multi-jet modes appear successively with the increase of applied voltage. In the absence of an electric field, the formation mechanism is that gravity overcomes the adhesion effect of surface tension to the capillary. The droplet size can be calculated theoretically. When voltage is applied, the surface tension of the charged liquid will be weakened by $\sigma=\sigma_{0}-q^{2} /\left(8 \pi^{2} \varepsilon D^{3}\right)$, where $\sigma$ and $\sigma_{0}$ are the surface tensions of charged and neutral droplets, $q$ is the charge density, $\varepsilon$ is the dielectric constant of the ambient medium, and $D$ is the droplet diameter. An electric field of $2.4 \mathrm{kV}$ decreases the droplet diameter significantly while increasing the dripping frequency (to approximately $93 \mathrm{~ms}$ ). Further increasing the voltage to $3.6 \mathrm{kV}$, the spray enters the cone-jet mode.

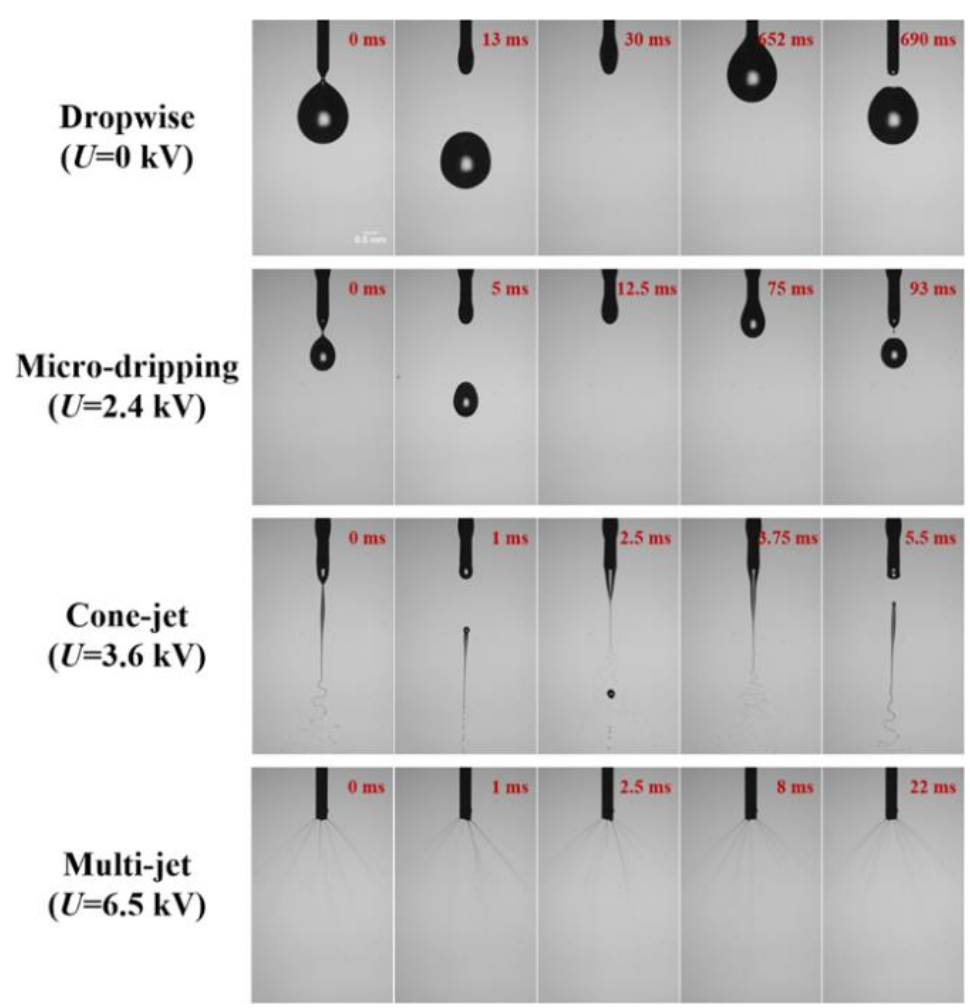

Figure 3. Dropwise, micro-dripping, cone-jet and multi-jet ES modes evolution at a flow rate of $20 \mathrm{ml} / \mathrm{h}$.

Spray characteristics of different ES modes are shown in Figure 4. Figure 4(a) displays the performance of spray size, which indicates that the droplet diameter goes down dramatically with applied voltage in the dripping mode, but levels off after reaching the jet modes. In addition, both dripping velocity and frequency are found to increase with applied voltage in Figure 4(b) and (c) owing to the increasing EHD force, especially during the transition from micro-dripping to cone-jet mode. However, the spray velocity and frequency of jet modes decrease with increased flow rate, which is because of the decreasing charge density for higher flow rate 
(same electric intensity but more liquid). Spray swath is defined as droplet diameter in dripping modes, while it is the diameter of the spray cone in jet modes. Figure 4(d) shows that spray swatch decreases in dripping modes but increases in jet modes with the increase of applied voltage.

Figure 5 shows the heat transfer performance of the four ES modes operated at a flow rate of $20 \mathrm{ml} / \mathrm{h}$. The spray captures of different modes are demonstrated in Figure 5(a). With the increase of applied voltage from $0 \mathrm{kV}$ to $6.5 \mathrm{kV}$, the atomization performance is found to be improved, as dropwise flow impacts only on the centre of the heated surface, while multi-jet fine spray covers most of the whole surface. Figure $5(\mathrm{~b})$ reveals the variation of heat flux with surface temperature. All four curves are shown to increase first before reaching an inflection point. However, there are some differences in ES cooling between modes. The behaviour of dropwise and micro-dripping modes is consistent with typical spray cooling [2]. The upper limit of heat flux of cone-jet ES cooling occurs at approximately $140^{\circ} \mathrm{C}$, and the cooling curve of multi-jet ES shows continuous increase as temperature increases. That is due to the fact that jet mode ES creates much smaller and faster droplets for spray heat transfer, and the image force pins the charged droplets on the hot surface, resulting in shorter evaporation time [6]. Theoretically, the image force results from the integration of the Maxwell stress $\tau_{\mathrm{e}}=\varepsilon_{0} E^{2} / 2$ at the gas-liquid interface, where $\varepsilon_{0}$ is the dielectric permittivity of surrounding medium, and $E$ represents the electric field at the interface. The magnitude of the image force can be estimated by a liquid spherical cap model and is about three orders larger than gravity for politer droplets. However, the effect of the image force on droplet impact behaviour only applies when there is still sufficient charge remaining on the droplet after impact. It means that the impact time $\left(\tau_{\mathrm{m}}=8 d / 3 v_{0}\right)$ should be less than the charge relaxation time $\left(\tau_{\mathrm{r}}=\varepsilon / K\right)$, where $d$ is the droplet diameter, $v_{0}$ is the impact velocity, $\varepsilon$ is the dielectric permittivity of the liquid, and $K$ is the electrical conductivity of the liquid.

(a)

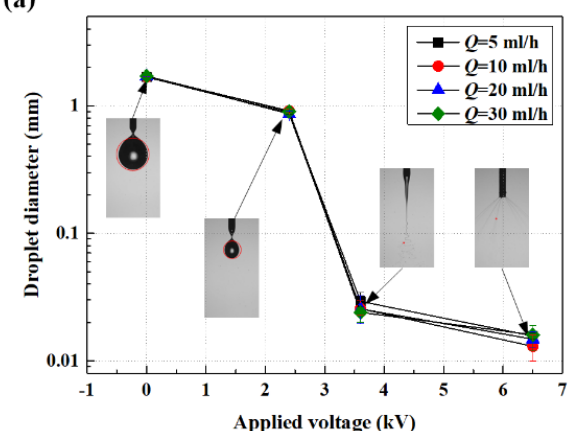

(c)

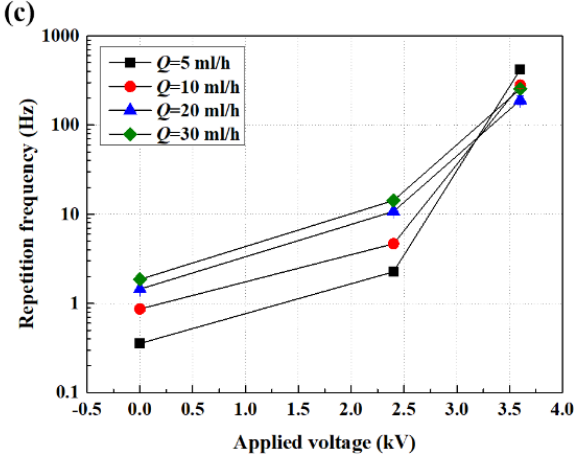

(b)

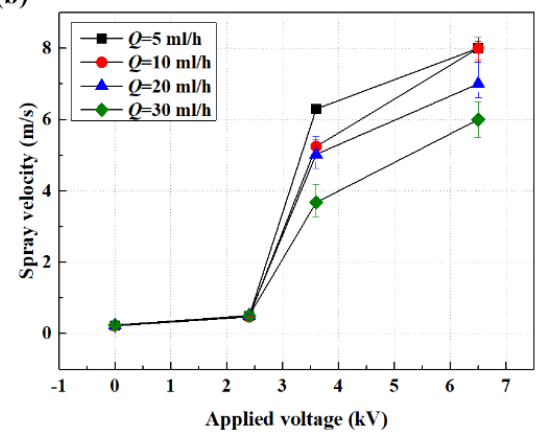

(d)

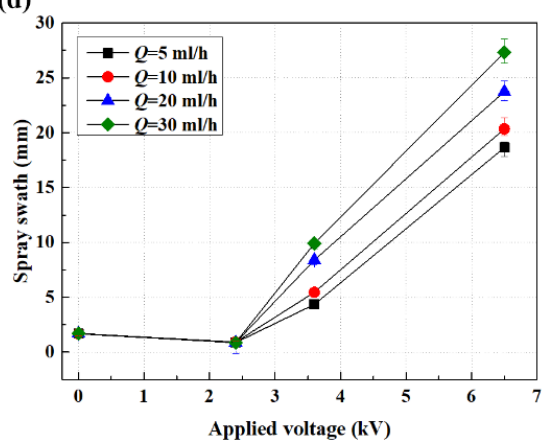

Figure 4. Spray characteristics of four modes ES, (a) droplets diameter, (b) spray velocity, (c) repetition frequency and (d) spray swath. 
The heat transfer coefficient performance is shown in Figure 5(c), where the heat transfer coefficient of the single-phase region is higher than that of the two-phase region, and ES cooling with a higher voltage has a greater heat transfer coefficient, especially at low surface temperatures. This is mainly attributed to the spreading of spray droplets caused by the electric force. Compared to neutral droplet, the charged one would show flattening effect on substrate due to surface tension decrease. Specifically, the contact angle of a sessile droplet has been reported to decrease from $81^{\circ}$ to $56^{\circ}$ after being charged [8]. Moreover, the maximum spreading diameter of charged impacting droplets has been found to be larger than that of neutral droplets [9]. Moreover, the variation of surface temperature shown in Figure 5(d) also indicates the active effect of electric filed on cooling enhancement.

(a)

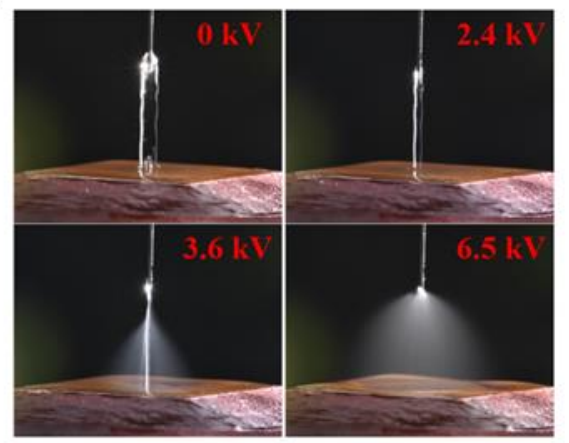

(c)

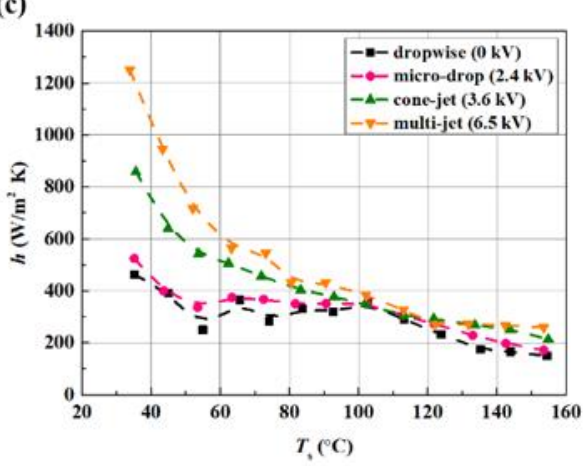

(b)

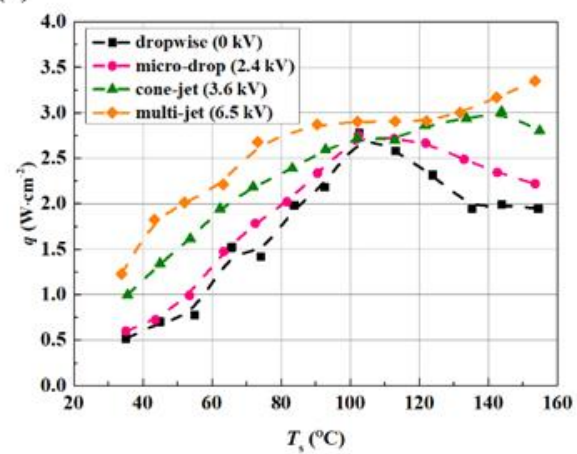

(d)

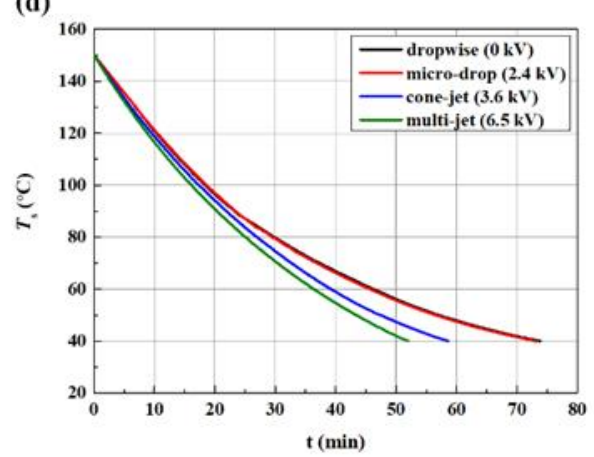

Figure 5. Cooling performance of four different modes ES, (a) spray morphology, (b) cooling curves, (c) heat transfer coefficient and (d) transient temperature variation.

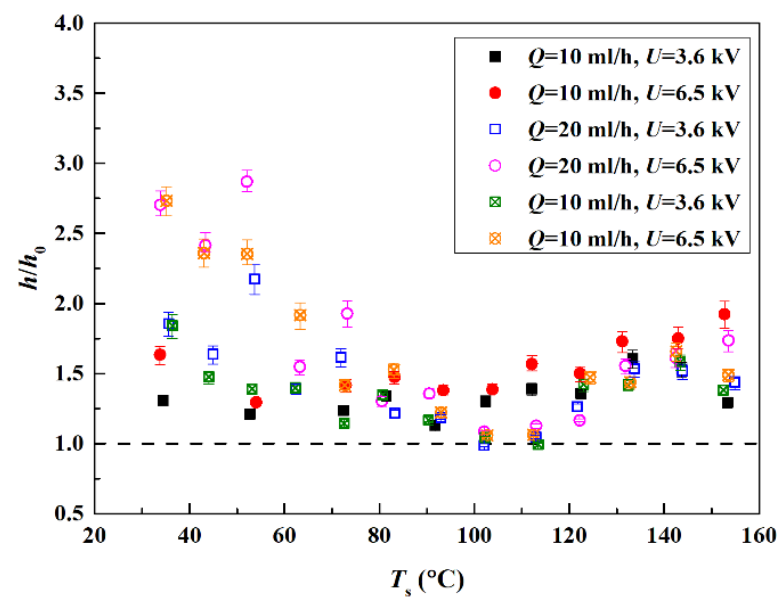

Figure 6. Variation of heat transfer enhancement ratio with the surface temperature for different conditions. 
To better reflect the effect from an electric field on cooling performance, the enhancement ratio $\left(h / h_{0}\right)$ is proposed, where $h$ is the convection heat transfer coefficient of ES cooling and $h_{0}$ is the convection heat transfer coefficient of neutral dropwise cooling with equivalent flow rates. The results are shown in Figure 6 . It could be found that the heat transfer coefficient is significantly improved varying with different surface temperatures. Specifically, for all the cases with different applied voltages and flow rates, the enhancement ratio is uniformly found to decrease first before gradually climbing up at about $110^{\circ} \mathrm{C}$ with increasing surface temperature. In this study, the heat transfer coefficient for ES cooling is improved up to 2.8 times, while only 1.7 times was reported in previous studies. This enhancing effect might be attributed to the droplet spreading improvement and the droplet rebound prevention caused by electrohydrodynamic effect for low-temperature and high-temperature conditions respectively, which will be discussed in detail in the next section.

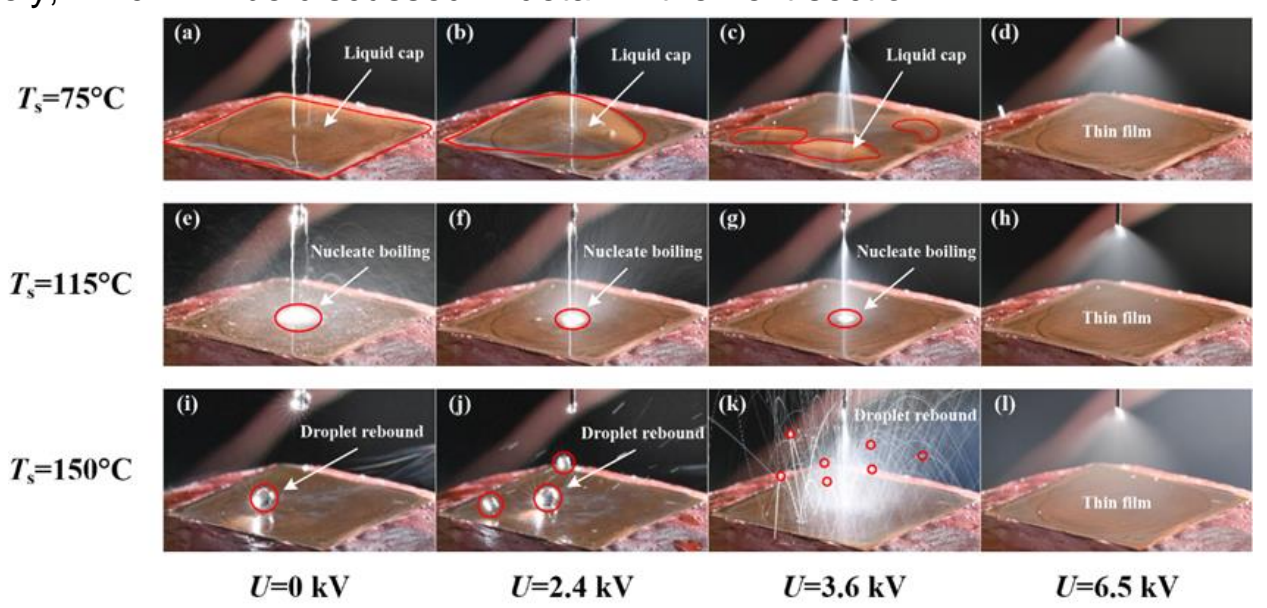

Figure 7. Dynamic behaviour and film formation of four modes of ES droplets for various surface temperature.

Spray cooling achieves high cooling capacity by transmitting coolant droplets with high velocity upon a hot surface. As was discussed, the applied voltage successfully influences the spray characteristics and improves the heat transfer performance. The droplets' impinging behaviour on surfaces has been investigated the determining factor of heat removal [10]. Figure 7 shows the impact behaviour and film formation of four modes of ES at various surface temperatures with a flow rate of $20 \mathrm{ml} / \mathrm{h}$. As shown in Figure 7(a), when the surface temperature is about $75^{\circ} \mathrm{C}$, the heating surface is completely covered with a heavy liquid film for the non-ES condition. However, the liquid cap becomes to partially cover the surface in Figure 7(b) while the liquid film on the heating surface is extremely thin and uniform in Figure 7(d). At the nucleate boiling point with surface temperature of about $115^{\circ} \mathrm{C}$, the impingement of spray droplets becomes more furious instead of forming a heavy "liquid cap." Most of the spray liquid evaporates in a very short time, as shown in Fig. 13(e) - (h). Further increasing the surface temperature to about $150^{\circ} \mathrm{C}$, the Leidenfrost phenomenon is clearly observed for dropwise and micro-dripping ES cooling in Figure 13(i) and (j). However, It should be noted that no rebound of impinging droplets is captured for multi-jet ES cooling over the testing temperature, and the heated surface maintains a very thin liquid film. This is how multi-jet ES can achieve outstanding cooling capacity.

\section{Conclusions}

In summary, the present work experimentally investigates the heat transfer performance of electrospray (ES) cooling with different spray modes. We explain differences in cooling 
characteristics by taking the spray characteristics and spray droplet impact behaviour into consideration. The mechanisms of ES are discussed, and four typical ES modes of ethanol, namely dropwise, micro-dripping, cone-jet, and multi-jet, are studied in this work. We find that as the spray mode shifts from dropwise/micro-dripping to cone-jet/multi-jet, the nucleate boiling region is significantly extended to higher surface temperatures. Specifically, a 2.8 times enhancement ratio of ES cooling is obtained compared to the neutral conditions. In addition, the dynamic behaviour of spray droplets impacting on a hot surface under different ES modes is comparatively studied. We found that the degrees of droplet spreading at the single-phase stage and spray droplet rebound in overheated conditions vary with spray modes, which contribute to the different cooling performances of these four ES modes. Undoubtedly, we have verified the importance of spray mode in ES cooling, which will promote the precise control of this enhancement technique.

\section{Acknowledgments}

The authors would like to thank the financial supports from the National Natural Science Foundation of China (No. 51976084, No. 52036007).

\section{References}

[1] Zhao, R., Cheng, W.-I., Liu, Q.-n., and Fan, H.-I., 2010, "Study on heat transfer performance of spray cooling: model and analysis," Heat and Mass Transfer, 46(8).

[2] Kim, J., 2006, "Spray cooling heat transfer: The state of the art," International Journal of Heat and Fluid Flow, 28(4).

[3] Cheng, W.-L., Zhang, W.-W., Chen, H., and Hu, L., 2016, "Spray cooling and flash evaporation cooling: The current development and application," Renewable and Sustainable Energy Reviews, 55.

[4] Taylor, G., 1964, "Disintegration of Water Drops in an Electric Field," Proceedings of the Royal Society of London. Series A, Mathematical and Physical Sciences (1934-1990), 280(1382).

[5] Xin, F., and E., B. J., 2008, "Application of Electrohydrodynamic Atomization to Two-Phase Impingement Heat Transfer," Journal of Heat Transfer, 130(7).

[6] Deng, W., and Gomez, A., 2011, "Electrospray cooling for microelectronics," International Journal of Heat and Mass Transfer, 54(11).

[7] Jaworek, A., and Sobczyk, A. T., 2007, "Electrospraying route to nanotechnology: An overview," Journal of Electrostatics, 66(3).

[8] Weiwei Deng, A. G., 2010, "The role of electric charge in microdroplets impacting on conducting surfaces," Physics of Fluids, 22, p. 051703.

[9] Ryu, S. U., and Lee, S. Y., 2008, "Maximum spreading of electrically charged droplets impacting on dielectric substrates," International Journal of Multiphase Flow, 35(1).

[10] L.H.J., W., L., S., J.R., V., and H.C., K., 1966, "The heat transfer from a hot wall to impinging mist droplets in the spheroidal state," Pergamon, 21(12). 